\title{
Highly-boosted dark matter and cutoff for cosmic-ray neutrinos through neu- trino portal
}

\author{
Wen Yin $^{1,2, *}$ \\ ${ }^{1}$ Institute of High Energy Physics, Chinese Academy of Sciences, Beijing 100049, China \\ ${ }^{2}$ Department of Physics, Korea Advanced Institute of Science and Technology, 291 Daehak-ro, Yuseong-gu, Daejeon 34141, Republic \\ of Korea **
}

\begin{abstract}
We study the cutoff for the cosmic-ray neutrino, set by the scattering with cosmic background neutrinos into dark sector particles through a neutrino portal interaction. We find that a large interaction rate is still viable, when the dark sector particles are mainly coupled to the $\tau$-neutrino, so that the neutrino mean free path can be reduced to be $O(10) \mathrm{Mpc}$ over a wide energy range. If stable enough, the dark sector particle, into which most of the cosmic-ray neutrino energy is transferred, can travel across the Universe and reach the earth. The dark sector particle can carry the energy as large as $O(\mathrm{EeV})$ if it originates from a cosmogenic neutrino.
\end{abstract}

\section{Introduction}

The cosmogenic neutrinos are known as a "guaranteed" flux of cosmic ray neutrinos, which are generated through photo-pion production, where the interaction is between ultra-high cosmic rays (UHECRs) and ambient photon backgrounds [1], This interaction was first proposed to set a cutoff for UHECRs and the cutoff as well as the UHECRs have already been observed [2, 3]. However the IceCube observatory, whose one of the purposes is to detect the cosmogenic neutrinos, has not yet observed neutrinos with energy $\gg 10^{6} \mathrm{GeV}$. The Glashow resonance around $6 \mathrm{PeV}$ is also not observed [4].

These non-observations may relate to the neutrality of the neutrino. In particular, the neutrinos can interact with a dark sector, whose participants can carry some hidden charge, so that the lightest particle is stable to explain the dark matter i.e. there could be a neutrino portal interaction relevant to dark matter $[5,6]$. It was pointed out that a cutoff for the cosmic-ray neutrino can be set through its scattering with cosmic background neutrinos $(\mathrm{C} v \mathrm{~B})$ into the dark sector particles through the neutrino-portal interaction by using an effective theory approach [7]. (For other models or other scattering processes affecting cosmic-ray neutrinos, see e.g. Refs [8-11].) Thus, the Universe could be opaque to the cosmic-ray neutrino.

Here we study the propagation of cosmic-ray neutrinos with Dirac neutrino portal interaction, where the right-handed neutrinos are Dirac-type particles. This is a renormalizable model. The reason we consider Dirac-type right-handed neutrinos rather than the Majorana-type is to suppress the lepton number violating effect. We show that there are viable parameter regions, especially for the $\tau$ neutrino $\left(v_{\tau}\right)$ portal interaction, that the cosmic-ray neutri-

\footnotetext{
*e-mail: yinwen@kaist.ac.kr

** After the talk, the affiliation was changed from IHEP to KAIST.
}

nos get scattered with the $\mathrm{C} v \mathrm{~B}$ into the particles in the dark sector before they reach the earth. As a result, the neutrino flux is transferred into the dark sector particles which relaxes the tension from the non-observations. In particular, there are viable parameter regions, that the neutrino flux is highly-suppressed, which might be difficult to be detected in the observatories. Instead, a large fraction of the viable region may be tested in collider experiments. Since the energy of the cosmic-ray neutrinos is transferred into the dark matter, there could be a flux of highly-boosted dark matter in the universe. They reach the earth instead of the cosmic-ray neutrinos which may be tested in various neutrino observatories.

\section{Dirac neutrino portal interaction}

Let us consider the following interaction with lepton number symmetry as one example.

$$
\delta \mathcal{L}_{\text {int }}=-\sum_{i=e, \mu, \tau}\left(y_{R i} H \bar{v}_{R i} \hat{P}_{L} L_{i}+\tilde{y}_{i} \bar{v}_{R i} \hat{P}_{R} \psi \phi_{i}\right)+\text { h.c. }
$$

Here, $H$ is the standard model (SM) Higgs boson and $L_{i}$ is the left handed lepton in flavor basis where $i=e, \mu, \tau$. $\psi, v_{R i}$ and $\phi_{i}$ are the right-handed (Dirac) neutrino, a Majorana fermion, and leptonic scalars, respectively. These Yukawa matrices are diagonalized without loss of generality.

The mass terms of these fields are given by

$\delta \mathcal{L}_{\text {mass }}=-\sum_{i=e, \mu, \tau}\left(m_{\phi i j}^{2} \phi_{i}^{\dagger} \phi_{j}+M_{R i j} \bar{v}_{R i} v_{R j}+\frac{1}{2} M_{\psi} \bar{\psi} \psi\right)+$ h.c.,

where we have defined the lepton number conserving mass squares $m_{\phi_{i j}}^{2}$, Dirac masses for the right-handed neutrinos 
$M_{R i j}$, and the Majorana mass term $M_{\psi}$ for fermion $\psi$. The symmetry allows the potentials for $\phi_{i}$ of the form,

$$
V=\frac{1}{4} \sum_{i, j, k, l} \lambda_{i j k l} \phi_{i}^{\dagger} \phi_{j}^{\dagger} \phi_{k} \phi_{l}+\sum_{i, j} \phi_{i}^{\dagger} \phi_{j}\left(\epsilon_{i j}|H|^{2}-\epsilon_{i j} v^{2}\right) .
$$

Here, $v \simeq 174 \mathrm{GeV}$ is the vacuum expectation value of the Higgs field. The first term lets a leptonic scalar decay into the lightest scalars in the dark sector, if kinematically allowed. We will assume $\epsilon_{i j}$ of the second term is negligible to define the neutrino portal interaction. Notice that this is rarely generated radiatively when $\tilde{y}_{i} y_{j}$ are small enough.

At the lepton number symmetric limit, the SM neutrinos are massless. With some small soft breaking terms for lepton number, such as $\tilde{M}_{R i j} \overline{\nu_{R i}^{c}} v_{R j}$, and $B_{i j} \phi_{i} \phi_{j}$, the neutrino masses are generated, through inverse seesaw mechanism [12], or radiatively [7]. In both cases, a $Z_{2}$ symmetry remains from the lepton number breaking. The $Z_{2}$ symmetry makes the lightest one of $\phi_{i}, \psi$ stable, and allows it to be dark matter. The small lepton number breaking term does not change the following discussion, and we will work on with the symmetric limit.

For simplicity, hereafter we will take

$$
m_{\phi i j}^{2}=\operatorname{diag}\left\{m_{\phi_{e}}^{2}, m_{\phi_{\mu}}^{2}, m_{\phi_{\tau}}^{2}\right\}, M_{R i j}=\operatorname{diag}\left\{M_{R_{e}}, M_{R_{\mu}}, M_{R_{\tau}}\right\} .
$$

As discussed later, the experimental constraints on the $v_{R e}$ and $v_{R \mu}$ are more severe than the ones for $\nu_{R \tau}$ in the interesting parameter ranges. Thus, we will assume $y_{R \mu}$ and $y_{R e}$ are negligible and focus on the interaction for $\tau$ sector for simplicity.

\section{Cutoff for neutrino flux}

Now, consider the impact of the portal interaction to the propagation of neutrino in the Universe.

The neutrino $\alpha$ (in mass eigen state) mixes with the $v_{R \tau}$, with mixing angle $\simeq U_{\tau} \times\left(U_{\mathrm{PNMS}}\right)_{\tau \alpha}$. Thus the cosmic-ray neutrino scatters with the $\mathrm{C} v \mathrm{~B}$ through the mixing,

$$
v_{\alpha}+v_{\beta}^{\mathrm{C} v \mathrm{~B}} / \bar{\nu}_{\beta}^{\mathrm{C} v \mathrm{~B}} \rightarrow \psi+\psi, \phi_{\tau}+\phi_{\tau} / \phi_{\tau}^{*} .
$$

The total scattering cross section with center of mass energy $E_{\mathrm{cm}}$ is given by

$\sigma_{\alpha \beta} v_{\mathrm{rel}} \simeq \theta\left(1-\frac{2 m_{\phi_{\tau}}}{E_{\mathrm{cm}}}\right) R_{\alpha \beta} \frac{1}{32 \pi E_{\mathrm{cm}}^{2}} \log \left(\frac{E_{\mathrm{cm}}+\sqrt{E_{\mathrm{cm}}^{2}-4 m_{\phi_{\tau}}^{2}}}{E_{\mathrm{cm}}-\sqrt{E_{\mathrm{cm}}^{2}-4 m_{\phi_{\tau}}^{2}}}\right)$

where we have defined

$$
R_{\alpha \beta}=\left|\left(U_{\mathrm{PNMS}}\right)_{\alpha \tau}\right|^{2}\left|\left(U_{\mathrm{PNMS}}\right)_{\beta \tau}\right|^{2}\left|U_{\tau}\right|^{4}\left|\tilde{y}_{\tau}\right|^{4}
$$

and assumed $M_{\psi}=m_{\phi_{\tau}}$ for illustrative purpose; $v_{\text {rel }}$ is the relative velocity between the two neutrinos at the center of mass frame; $U_{i} \equiv \frac{y_{i} v}{M_{R i}}$ is the mixing angle between the left and right handed neutrinos; We have taken the average for the target neutrino and anti-neutrino for the crosssection;

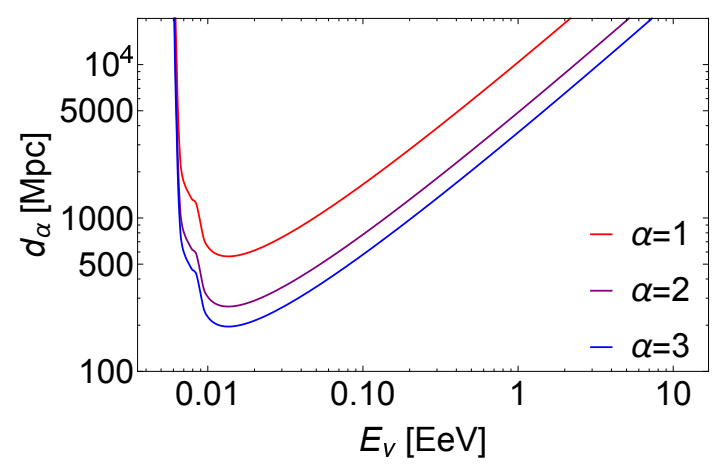

Figure 1. The mean free path for the neutrino in mass eigenstate $\alpha$ as a function of energy. We take $M_{\psi}=m_{\phi_{\tau}}=15 \mathrm{MeV}$ and $\left|U_{\tau} \tilde{y}_{\tau}\right|=0.4$.

The unit stepfunction $\theta$ represents the momentum conservation. The energy of the cosmic ray $E_{v}$ in our frame is related with the center of mass energy as

$$
E_{\mathrm{cm}} \simeq \sqrt{2\left(\sqrt{|\mathbf{p}|^{2}+m_{\nu \alpha}} E_{\nu}-|\mathbf{p}| E_{\nu} \cos \theta\right)}
$$

with neutrino mass $m_{v \alpha}$ and momentum $\mathbf{p}$ of a $\mathrm{C} v \mathrm{~B}$ neutrino.

The interaction rate of the neutrino $v_{\alpha}$ is obtained by taking the thermal average of the previous cross section,

$$
\Gamma_{\alpha}\left(E_{v}, T_{v}\right)=\sum_{\beta}\left\langle\sigma_{\alpha \beta} v_{\text {rel }} n_{v \beta}\right\rangle
$$

Here, $n_{v \alpha}(\mathbf{p})=2 /\left(e^{|\mathbf{p}| / T_{v}}+1\right)$ is the neutrino distribution in $\mathrm{C} v \mathrm{~B}$. \langle\rangle denotes the thermal average. The mean free path of neutrino is defined by

$$
d_{\alpha}\left(E_{v}, T_{v}\right) \equiv \frac{1}{\Gamma_{\alpha}\left(E_{v}, T_{v}\right)} .
$$

The numerical result for $d_{\alpha}\left(E_{v}, T_{v}^{\text {now }}\right)$ is presented Fig. 1, where $T_{v}^{\text {now }} \simeq 1.69 \times 10^{-4} \mathrm{eV}$ is the current temperature of the $\mathrm{C} v \mathrm{~B}$. Here and hereafter, we take the normal mass ordering case with lightest neutrino mass $0.05 \mathrm{eV}$ and the Dirac phase $\delta_{13}=-\pi / 2$. The numerical result can be approximately read as

$$
\Gamma_{\alpha} \sim(1 \mathrm{Gpc})^{-1}\left|\frac{\tilde{y}_{\tau} U_{\tau}}{0.3}\right|^{4}\left(\frac{300 \mathrm{MeV}}{E_{\mathrm{cm}}}\right)^{2} \theta\left(E_{\mathrm{cm}}-m_{\phi_{\tau}} / 2\right) .
$$

The stepfunction implies that the mean free path of neutrino with

$$
E_{v} \gtrsim \frac{m_{\phi_{\tau}}^{2}}{\max \left\{m_{v \text { lightest }}, T_{v}\right\}}
$$

becomes smaller than $O(\mathrm{Gpc})$, when

$$
\left|\tilde{y}_{\tau} U_{\tau}\right| \gtrsim O(0.1) .
$$

By giving the neutrino masses of $O(0.001-0.1) \mathrm{eV}$, this implies that a neutrino with $E_{v}=10^{6-10} \mathrm{GeV}$ is possible to be scattered before it travels over $O(1) \mathrm{Gpc}$ for 


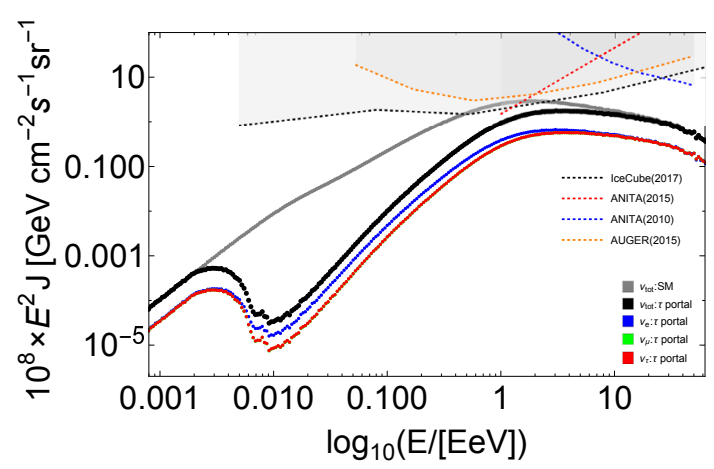

Figure 2. The flux $\times E^{2}$ for the total neutrinos (black), $v_{e}$ (blue), $v_{\mu}$ (Green), $v_{\tau}$ (red) as a function of energy. For comparison, flux $\times E^{2}$ of total neutrinos within the SM is also shown (gray). We take $M_{\psi}=m_{\phi_{\tau}}=15 \mathrm{MeV}$ and $\left|U_{\tau} \tilde{y}_{\tau}\right|=0.4$. The constraints (shaded regions) at 90\% CL are adapted from [14-17].

$m_{\phi_{\tau}} \simeq O(10-100) \mathrm{MeV} .{ }^{1}$ This can give an explanation of the non-observation of cosmogenic neutrino as well as the Glashow resonance.

Now let us perform a numerical estimation on the neutrino flux at the earth. We assume an original cosmogenic neutrino flux and that the produced $\phi_{\tau}, \phi_{\tau}^{*}$ and $\psi$ does not lead to energetic secondary neutrinos for the moment. This might be the case that $\phi_{\tau}, \phi_{\tau}^{*}$ and $\psi$ soon decay into the other dark sector fields. A case with secondary neutrinos will be studied in the next section.

The number density of the cosmogenic neutrinos are produced at a rate $n_{v_{e}}: n_{v_{\mu}}: n_{v_{\tau}} \simeq 1: 2: 0$ of flavor through photo-pion interaction. The number density in the mass basis is given by $n_{v_{\alpha}}=\sum_{i}\left(U_{\mathrm{PNMS}}^{*}\right)_{\alpha i}\left(U_{\mathrm{PNMS}}\right)_{\alpha i} n_{v_{i}}$ and $n_{v_{1}}: n_{v_{2}}: n_{v_{3}} \sim 1: 1: 1$. Since the free propagating length $d_{\alpha} \gtrsim \mathcal{O}(\mathrm{Mpc})$ is much longer than the neutrino oscillation scale, the interference between different neutrinos in mass basis disappears due to the rapid oscillation. As a result, the neutrinos travel in the mass basis with the ratio $\sim 1$ : $1: 1$ kept.

When the traveling distance becomes $\gtrsim d_{\alpha}$ the neutrino flux is gradually transferred into the dark sector through scattering between the neutrino and $\mathrm{C} v \mathrm{~B}$. A neutrino $\alpha$ emitted at red shift $z=z_{s}$ travels until now at a survivability of,

$$
R_{\alpha}\left(E_{\mathrm{now}}, z_{S}\right)=e^{-\int_{0}^{z s} d z\left|\frac{d t}{d z}\right| \Gamma_{\alpha}\left(E_{\mathrm{now}}(1+z), T_{v}(1+z)\right)},
$$

where $E_{\text {now }}$ is the energy measured in the current universe, and we have taken account of the effect for the redshift for the energy and $T_{v}$. Here $\left|\frac{d t}{d z}\right| \equiv$ $\left((1+z) \sqrt{\Omega_{M}(1+z)^{3}+\Omega_{\Lambda}}\right)^{-1}$. Thus, the neutrino in a flavor basis $i$ reaches the earth with a probability,

$$
\tilde{R}_{i}=R_{\alpha}\left(E_{\mathrm{now}}, z_{s}\right)\left|\left(U_{\mathrm{PNMS}}\right)_{i \alpha}\right|^{2} .
$$

Using CRPropa 3 [13] we have made a numerical simulation on the cosmogenic neutrino source, assuming

\footnotetext{
1 The lower bound of $m_{\phi \tau}$ is from the cosmological constraints on dark radiation.
}

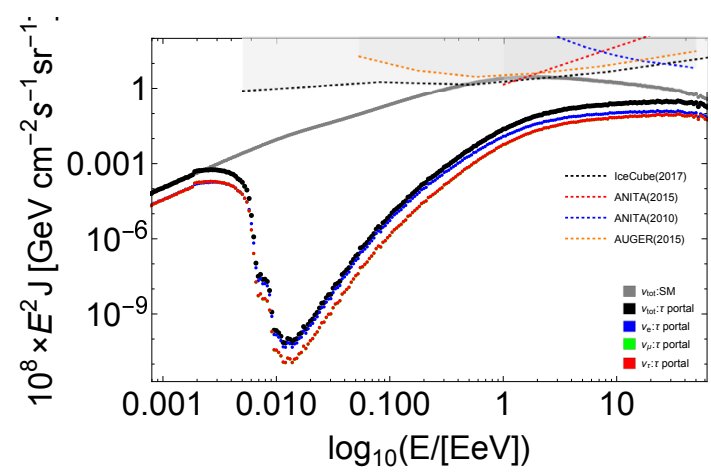

Figure 3. Same as Fig. 2, but $\left|U_{\tau} \tilde{y}_{\tau}\right|=1$.

the observed UHECR are purely protons. For each cosmogenic neutrino, we calculate the survivability (14) from its profile given by CRPropa 3. As a result, we obtain the neutrino flux as in Figs. 2 and 3 for the effective coupling $\left|\tilde{y}_{\tau} U \tau\right|=0.4$ and 1 , respectively. In the numerical simulation, we use one of the best fit parameter given in Ref. [18]: the proton source of energy $E_{p}$ is set to have a power law distribution $\propto\left(E_{p}\right)^{-2.49}$ between $10^{17.5} \mathrm{eV}<E_{p}<10^{21} \mathrm{eV}$ for $z<z_{\max }=2$, the cosmic evolution rate is represented by $(1+z)^{3.5}$ until $z=z_{\max }$. Also shown is the current bound from the experiments of IceCube, AUGER, and ANITA. We found that the cosmogenic neutrino flux to the earth can be significantly reduced due to the neutrino portal interaction.

In fact, there are allowed regions for the $v_{\tau}$ portal interaction with $\left|\tilde{y}_{\tau} U_{\tau}\right| \lesssim O(1)$. Furthermore, a large portion of the allowed region can be tested in future from collider experiments. In Figs. 4 and 5 we show the viable/testable parameter region for this model, and the mean free path at the right hand side. When $v_{R \tau}$ is heavier than $\tau$, it only decays into lighter mass eigenstate with weak current ratio $\frac{g_{\tau}}{g_{\mu, e}}=\sqrt{1-\left|U_{\tau}\right|^{2}}$. The constraints on the ratio are given by $\frac{g_{\tau}}{g_{\mu}}=1.0001 \pm 0.0014$ and $\frac{g_{\tau}}{g_{e}}=1.0029 \pm 0.0015$ [19], and we have combined them to find the lepton universality bound (orange band)

$$
\left|U_{\tau}\right| \lesssim 0.0027(99 \% \mathrm{CL})\left(M_{R \tau}>m_{\tau}\right) .
$$

When $M_{R \tau}$ is much smaller than $m_{\tau}$, the neutrino in the decay product is represented by the flavor eigenstate and the lepton universality bound does not apply. When $M_{R \tau}$ is slightly lighter than the $\tau$ lepton, the kinematics of the visible decay products of $\tau$ are different, which could be distinguished. This indirectly constrains the parameter region (Red band: 95\%CL limit) [20, 21]. In particular, it was discussed in Ref. [21], the future B-factories could test the scenario with kinematic measurement of semi-leptonic $\tau$ decay (red solid line: conservative, red dashed line: optimistic).

The Higgs boson can decay into neutrinos and the dark sector fields or the right-handed neutrino. Such decay enhances the branching ratio of the Higgs boson to missing energy. The contribution to the decay rate of our scenario 


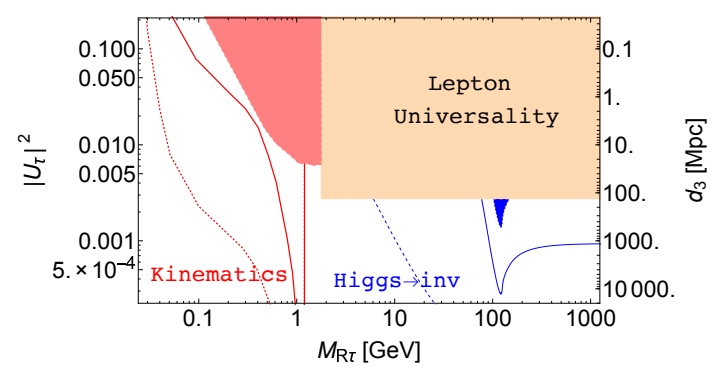

Figure 4. The viable region for $\tau$-neutrino portal interaction. The mean free path of the heaviest neutrino with $E_{v}=1 \mathrm{EeV}$ is given in the right-hand side. We have taken $M_{R \tau}>m_{\phi_{\tau}} \simeq M_{\psi}=$ $15 \mathrm{MeV}$ and $\tilde{y}_{\tau}=4 \pi$. The colored region might be excluded.

can be calculated as,

$$
\begin{aligned}
\Gamma_{H \rightarrow \text { missing }} & \simeq \frac{\left|y_{R \tau}\right|^{2}}{16 \pi} m_{h}\left(1-\frac{\left|M_{R \tau}\right|^{2}}{m_{h}^{2}}\right)^{2} \theta\left(1-\left|M_{R \tau}\right| / m_{h}\right) \\
& +\frac{\left|\tilde{y}_{\tau}^{2} y_{R \tau}^{2}\right|}{512 \pi^{3} m_{h}{ }^{3}}\left(2 M_{R \tau}^{2}\left(\left|M_{R \tau}\right|^{2}-m_{h}^{2}\right) \log \left(\left|\frac{m_{h}^{2}}{M_{R \tau}^{2}}-1\right|\right)\right. \\
& \left.-m_{h}{ }^{4}+2 m_{h}{ }^{2} M_{R \tau}^{2}\right) .
\end{aligned}
$$

The tree contribution for the process $H \rightarrow v_{\tau}+\bar{v}_{R \tau}$ is given in the first row. In particular, we have included the process $H \rightarrow v_{\tau}+\phi_{\tau}+\psi$, which becomes important when $M_{R \tau}>m_{h}$. We have made an analytical continuation in the calculation of the three-body decay so that the leading loop correction for the two-body decay is also included. We have neglected the mass of $\phi_{\tau}$ and $\psi$, whose interesting range for us is much smaller than the Higgs boson mass. When $4 \lesssim \tilde{y}_{\tau} \lesssim 4 \pi$, which may suggest $\phi_{\tau}$ is like a pion, the region affecting the cosmic-ray neutrino propagation may also be tested from the Higgs boson decay in the $\mathrm{LHC}$ which is proposed to measure the branching ratio at a precision of 0.05 (blue solid line) [22]. ${ }^{2}$ Future lepton colliders may reach $\sim 0.001$ (blue-dashed line) [25-29]. ${ }^{3}$

Notice that we have taken the mass range that $v_{R \tau}$ decays into $\phi_{\tau}$ and $\psi$. In the case where the decay channel is forbidden, $v_{R \tau}$ could have a much longer lifetime and decay into the SM particles. This leads to more severe constraints from beam dump experiments, as well as cosmology (see Refs. [31, 32] ). However, there is still an allowed region at $M_{R \tau} \simeq O(10) \mathrm{MeV}$ to affect the cosmicray neutrino flux.

When the neutrinos portal is through $v_{\mu}$ or $v_{e}$ the experiment constraints becomes more severe [6]. The measurement on meson decays sets stringent constraints and almost excludes all the viable region supressing the cosmicray neutrino with $M_{R i} \lesssim m_{K} \sim 500 \mathrm{MeV}$. Above the kaon mass, there are constraints from lepton universality as the $\tau$ case. When $M_{R i} \gtrsim m_{K}$ with $\tilde{y}_{i}>O(1)$, there are still viable regions. The prediction for the Higgs boson decay, which we have calculated, holds.

\footnotetext{
${ }^{2}$ Blue shaded region may be excluded [23, 24].

${ }^{3}$ We note that $\left|U_{\tau}\right|<0.42$ from the $\tau-\mu$ neutrino oscillation with matter effects [30].
}

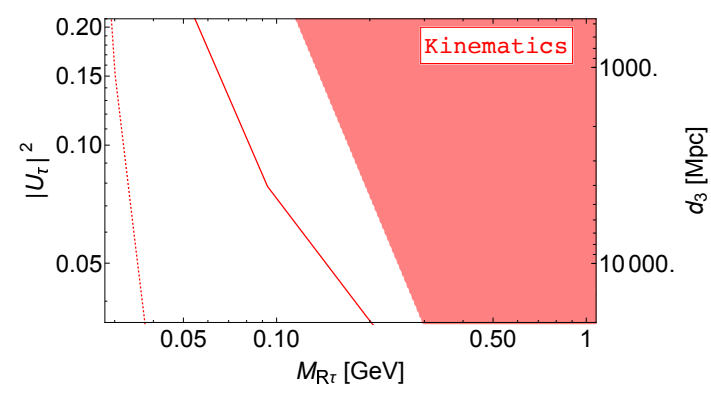

Figure 5. Same as Fig. 4 except for $\tilde{y}_{\tau}=1$.

\section{Highly boosted dark matter}

Since $\phi_{i}$ and $\psi$ are the only $Z_{2}$ odd particles, the lightest one among them is stable and could be the dark matter. This gives a distinguishable and interesting prediction of our scenario: the highly-boosted dark matter whose energy originates from the cosmogenic neutrinos. There are two cases for this scenario: (a) dark matter is directly produced through interaction (5), (b) dark matter is produced through the cascade decay of $\phi_{\tau}$ and $\psi$. The discussion in the previous section can correspond to case (b), where the decay products are assumed to rarely interact with $\mathrm{C} v \mathrm{~B}$ and without introducing secondary energetic neutrinos.

Now let us consider case (a). For simplicity, suppose that $0<M_{\psi}-m_{\phi_{\tau}} \ll m_{\phi_{\tau}}$, and $\phi_{e}$ and $\phi_{\mu}$ are much heavier. In this case, the produced dark matter, $\phi_{\tau}$, has energy $\sim$ $E_{v} / 2$ (or the produced $\psi$ decays to $\phi_{\tau}$ with energy $E_{v} / 2$ and a soft $\tau$ neutrino.). The dark matter travels across the universe for a long distance. It loses energy dominantly through the scattering with the $\mathrm{C} v \mathrm{~B},{ }^{4}$

$$
\phi_{\tau}+v_{\tau}^{\mathrm{C} v \mathrm{~B}} / \bar{\nu}_{\tau}^{\mathrm{C} v \mathrm{~B}} \rightarrow \phi_{\tau}+v_{\alpha} / \bar{v}_{\alpha}
$$

Since this is an elastic scattering process, the energy-loss rate becomes more important than the interaction rate. The energy loss rate for the interaction is given by

$$
\begin{aligned}
& \Gamma_{\mathrm{DM}, \alpha} \equiv \frac{d}{d t} \log E \\
& \simeq\left|\left(U_{\mathrm{PNMS}}\right)_{\tau \alpha}\right|^{2} \sum_{\beta=1,2,3}\left\langle\left.\sigma_{\phi_{\tau} \beta} v_{\mathrm{rel}} n_{\nu \beta}\left(1-\frac{E_{\mathrm{DM}}^{\mathrm{f}}}{E_{\mathrm{DM}}^{\mathrm{i}}}\right)||\left(U_{\mathrm{PNMS}}\right)_{\tau \beta}\right|^{2} .\right.
\end{aligned}
$$

where $E_{\mathrm{DM}}^{\mathrm{i}}$ and $E_{\mathrm{DM}}^{\mathrm{f}}$ are the energies of the dark matter in the initial and final states; $\sigma_{\sigma_{\phi_{\tau} \alpha}}$ is the scattering crosssection of (18). The energy loss length is given by

$$
d_{\mathrm{DM}} \equiv \frac{1}{\sum_{\alpha} \Gamma_{\mathrm{DM}, \alpha}} .
$$

This is shown in the Fig. 6. $d_{\mathrm{DM}}$ is slightly longer than $d_{\alpha}$ at $E_{\mathrm{cm}} \gg m_{\phi_{\tau}}, M_{\psi}$. This is due to the helicity suppression. Analytically one finds

$$
\Gamma_{\mathrm{DM}, \alpha} \sim \frac{1}{3}\left(1+\log \left(\frac{E_{\mathrm{cm}}^{2}}{\left|M_{\psi}\right|^{2}}\right)\right)^{-1} \Gamma_{\alpha} .
$$

\footnotetext{
${ }^{4}$ We have assumed that the right-handed neutrino is heavy enough that the process to $\phi_{\tau}+v_{\tau_{R}}$ is forbidden.
} 


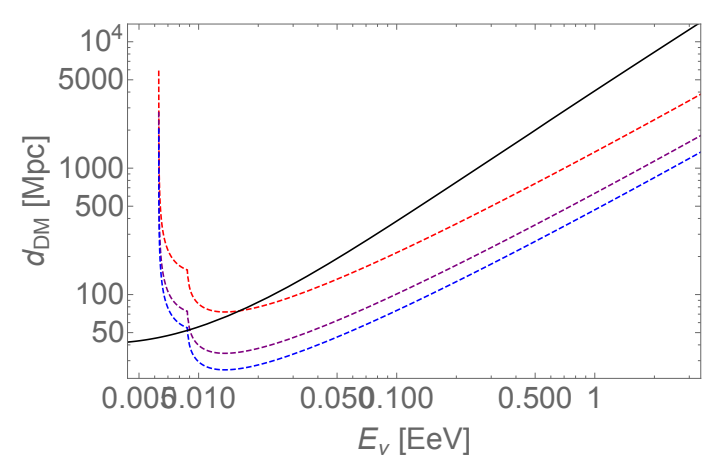

Figure 6. The energy loss length for dark matter (black solid). $m_{\phi_{\tau}} \simeq M_{\psi}=15 \mathrm{MeV}$ and $\left|\tilde{y}_{\tau} U_{\tau}\right|=0.5$ are taken. For comparison the mean free path of neutrinos are also shown (see Fig. 1).

which implies a suppression of several factors for $\Gamma_{\mathrm{DM}, \alpha}$ compared with $\Gamma_{\alpha}$. As a result, the dark matter at high energy produced through Eq.(18) is easier to reach the earth than a cosmogenic neutrino.

We perform a numerical simulation on the evolution of the number density of neutrinos and produced dark matter. The differential equation is given by,

$$
\begin{aligned}
\frac{\partial}{\partial t} J_{v \alpha}\left(E_{v}\right) & \simeq H(z) \frac{\partial}{\partial E_{v}}\left(E_{v} J_{v \alpha}\right)-\Gamma_{\alpha} J_{v \alpha}+J_{\text {source }} \\
& +\int d E_{\phi_{\tau}} \frac{d \sigma_{\phi_{\tau} \alpha}}{d E_{v}} v_{\text {rel }} n_{\mathrm{C} v \mathrm{~B}} J_{\phi_{\tau}}\left(E_{\phi_{\tau}}\right), \\
\frac{\partial}{\partial t} J_{\phi_{\tau}}\left(E_{\phi_{\tau}}\right) & \simeq H(z) \frac{\partial}{\partial E_{\phi_{\tau}}}\left(E_{\phi_{\tau}} J_{\phi_{\tau}}\right)-\Gamma_{\phi_{\tau}} J_{\phi_{\tau}} \\
& +\int d E_{v} \sum_{\alpha} \frac{d \sigma_{\alpha}}{d E_{\phi_{\tau}}} v_{\mathrm{rel}} n_{\mathrm{C} v \mathrm{~B}} J_{v \alpha}\left(E_{v}\right) \\
& +\int d \tilde{E}_{\phi_{\tau}} \sum_{\alpha} \frac{d \sigma_{\phi_{\tau} \alpha}}{d E_{\phi_{\tau}}} v_{\mathrm{rel}} n_{\mathrm{C} v \mathrm{~B}} J_{\phi_{\tau}}\left(\tilde{E}_{\phi_{\tau}}\right),
\end{aligned}
$$

Here, $J_{\ldots} \equiv \frac{\partial n_{\ldots .}}{\partial \log E} ; H(z)$ is the Hubble parameter at $z$; $\Gamma_{\phi_{\tau}}\left(E_{\nu}, T_{\nu}\right)=\sum_{\beta}\left\langle\sigma_{\phi_{\tau} \beta} v_{\text {rel }} n_{\nu \beta}\right\rangle ; J_{\text {source }}$ represents the cosmogenic neutrino number density emitted at red shift $z(t)$, which is fitted from CRPropa 3; for simplicity we have assumed that the neutrinos and anti-neutrinos are in the same distribution. The terms with integrals represent the re-scattering process. In the equation, $E$ and $T_{v}$ are related to the current value by $E=E_{\text {now }}(1+z(t))$ and $T_{v}=(1+z(t)) T_{v}^{\text {now }}$, at the time $t$. The numerical result is given in Fig. 7 for $\left|\tilde{y}_{\tau} U_{\tau}\right|=0.5$. One finds that the peak flux of dark matter can be as large as the one for the original cosmogenic neutrino for $E=O\left(10^{9}\right) \mathrm{GeV}$.

\section{Discussion}

The highly boosted dark matter scatters with a nucleon as $\phi_{\tau}+N \rightarrow \psi+\tau / v_{\tau}+N$. The cross section is of order, $\sigma_{\phi_{\tau} N} \sim \frac{1}{16 \pi^{2}}\left|\tilde{y} U_{\tau}\right|^{2} \times \sigma_{v_{\tau} N}$ where $\sigma_{v \tau N}$ is the $v_{\tau}-N$ scattering cross section of $v_{\tau}+N \rightarrow \tau+N$ in SM and $\frac{1}{16 \pi^{2}}$ represents the phase space suppression. Since this is $O\left(10^{-3}\right)-O\left(10^{-1}\right)$ suppressed to $\sigma_{v_{\tau} N}$, Since the earth is much more transparent for the dark matter than for a SM neutrino, the events for the highly boosted dark matter

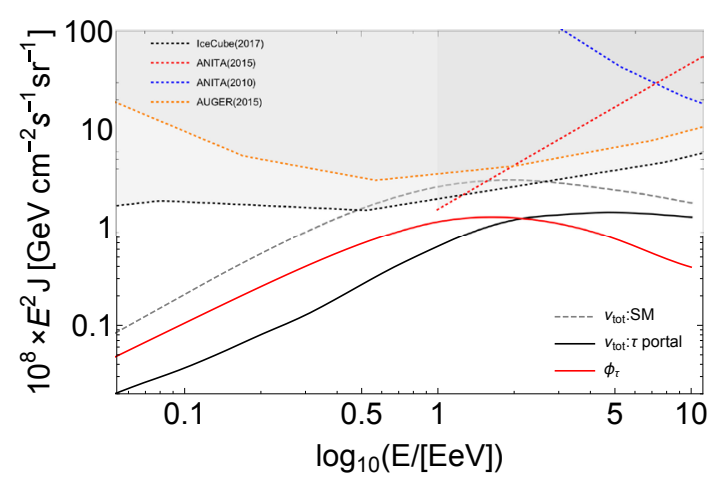

Figure 7. The predicted fluxes of neutrinos (black solid line) and dark matter (red solid line). $m_{\phi_{\tau}} \simeq M_{\psi}=15 \mathrm{MeV}$ and $\left|\tilde{y}_{\tau} U_{\tau}\right|=0.5$ are taken.

can be distinguished from the ordinary cosmogenic neutrinos from how long it travels within the earth. It may be tested or might be already detected in the ANITA experiment (c.f. Refs. [33, 34]).

The coupling of $\left|\tilde{y}_{\tau} U_{\tau}\right|=O(0.1)$ suggests a too large annihilation cross section for $\phi_{\tau}$ to get a correct thermal relic abundance. One needs another dominant dark matter candidate..$^{5} \psi$, if is extended into a Dirac fermion, can be asymmetric dark matter [5]. Interestingly, the parameter region for addressing small-scale structure issues also has $y_{\tau} U_{\tau}=O(0.1)$ and $m_{\mathrm{DM}}=O(10-100) \mathrm{MeV}$ [6], which may be coincident with the parameter region suppressing cosmic-ray neutrinos and highly boosted dark matter. The clarification of the coincidence will be our future study.

\section{Conclusions}

We have shown that a cutoff for the cosmic-ray neutrino can be set by the scattering with cosmic background neutrinos through a neutrino portal interaction. In particular, a large interaction rate is still allowed for $\tau$-neutrino portal interaction which is being or to be tested in the ongoing/future collider experiments. Highly-boosted dark matter can reach the earth instead of the cosmic-ray neutrinos and may be tested in the near future and might be already detected in the ANITA experiment.

\section{References}

[1] V. S. Berezinsky and G. T. Zatsepin, Phys. Lett. 28B, 423 (1969).

[2] K. Greisen, Phys. Rev. Lett. 16, 748 (1966).

[3] G. T. Zatsepin and V. A. Kuzmin, JETP Lett. 4, 78 (1966) [Pisma Zh. Eksp. Teor. Fiz. 4, 114 (1966)].

[4] S. L. Glashow, Phys. Rev. 118, 316 (1960).

[5] D. E. Kaplan, M. A. Luty and K. M. Zurek, Phys. Rev. D 79, 115016 (2009) [arXiv:0901.4117 [hep$\mathrm{ph}]]$.

[6] B. Bertoni, S. Ipek, D. McKeen and A. E. Nelson, JHEP 1504, 170 (2015) [arXiv:1412.3113 [hep-ph]].

\footnotetext{
${ }^{5}$ This may be the inflaton (e.g. Refs. $[35,36]$ ) or some superpartners (e.g. Refs.[37-41]).
} 
[7] W. Yin, arXiv:1706.07028 [hep-ph].

[8] K. Ioka and K. Murase, PTEP 2014, no. 6, 061E01 (2014) [arXiv:1404.2279 [astro-ph.HE]].

[9] J. F. Cherry, A. Friedland and I. M. Shoemaker, arXiv:1411.1071 [hep-ph].

[10] K. C. Y. Ng and J. F. Beacom, Phys. Rev. D 90 (2014) no.6, 065035 Erratum: [Phys. Rev. D 90 (2014) no.8, 089904] [arXiv:1404.2288 [astro-ph.HE]].

[11] M. Ibe and K. Kaneta, Phys. Rev. D 90, no. 5, 053011 (2014) [arXiv:1407.2848 [hep-ph]].

[12] R. N. Mohapatra and J. W. F. Valle, Phys. Rev. D 34, 1642 (1986).

[13] R. Alves Batista et al., JCAP 1605, no. 05, 038 (2016) [arXiv:1603.07142 [astro-ph.IM]].

[14] H. Schoorlemmer et al., Astropart. Phys. 77, 32 (2016) [arXiv:1506.05396 [astro-ph.HE]].

[15] M. G. Aartsen et al. [IceCube Collaboration], arXiv:1710.01191 [astro-ph.HE].

[16] A. Aab et al. [Pierre Auger Collaboration], Phys. Rev. D 91, no. 9, 092008 (2015) [arXiv:1504.05397 [astro-ph.HE]].

[17] P. W. Gorham et al. [ANITA Collaboration], Phys. Rev. D 82, 022004 (2010) Erratum: [Phys. Rev. D 85, 049901 (2012)] [arXiv:1003.2961 [astro-ph.HE], arXiv:1011.5004 [astro-ph.HE]].

[18] M. Ahlers, L. A. Anchordoqui, M. C. GonzalezGarcia, F. Halzen and S. Sarkar, Astropart. Phys. 34, 106 (2010) [arXiv:1005.2620 [astro-ph.HE]].

[19] A. Lusiani [BaBar Collaboration], EPJ Web Conf. 118, 01018 (2016).

[20] J. C. Helo, S. Kovalenko and I. Schmidt, Phys. Rev. D 84, 053008 (2011) [arXiv:1105.3019 [hep-ph]].

[21] A. Kobach and S. Dobbs, Phys. Rev. D 91, no. 5, 053006 (2015) [arXiv:1412.4785 [hep-ph]].

[22] M. E. Peskin, arXiv:1312.4974 [hep-ph].

[23] G. Aad et al. [ATLAS Collaboration], JHEP 1511, 206 (2015) [arXiv:1509.00672 [hep-ex]].
[24] V. Khachatryan et al. [CMS Collaboration], JHEP 1702, 135 (2017) [arXiv:1610.09218 [hep-ex]].

[25] CEPC-SPPC Study Group, http://cepc.ihep.ac.cn/preCDR/main_preCDR.pdf

[26] CEPC-SPPC Study Group, http://cepc.ihep.ac.cn/preCDR/PreCDR_final_20150317.pdf

[27] D. M. Asner et al., arXiv:1310.0763 [hep-ph].

[28] D. d'Enterria, arXiv:1602.05043 [hep-ex].

[29] H. Abramowicz et al., arXiv:1608.07538 [hep-ex].

[30] K. Abe et al. [Super-Kamiokande Collaboration], Phys. Rev. D 91, 052019 (2015) [arXiv:1410.2008 [hep-ex]].

[31] J. Orloff, A. N. Rozanov and C. Santoni, Phys. Lett. B 550, 8 (2002) [hep-ph/0208075].

[32] P. Astier et al. [NOMAD Collaboration], Phys. Lett. B 506, 27 (2001) [hep-ex/0101041].

[33] P. W. Gorham et al. [ANITA Collaboration], arXiv:1803.05088 [astro-ph.HE].

[34] J. F. Cherry and I. M. Shoemaker, arXiv:1802.01611 [hep-ph].

[35] R. Daido, F. Takahashi and W. Yin, "The ALP miracle: unified inflaton and dark matter," JCAP 1705, no. 05, 044 (2017) [arXiv:1702.03284 [hep-ph]].

[36] R. Daido, F. Takahashi and W. Yin, "The ALP miracle revisited," JHEP 1802, 104 (2018) [arXiv:1710.11107 [hep-ph]].

[37] Y. Shimizu and W. Yin, Phys. Lett. B 754, 118 (2016) [arXiv:1509.04933 [hep-ph]].

[38] W. Yin and N. Yokozaki, Phys. Lett. B 762, 72 (2016) [arXiv:1607.05705 [hep-ph]].

[39] T. T. Yanagida, W. Yin and N. Yokozaki, JHEP 1609, 086 (2016) [arXiv:1608.06618 [hep-ph]].

[40] W. Yin, Chin. Phys. C 42, no. 1, 013104 (2018) [arXiv:1609.03527 [hep-ph]].

[41] T. T. Yanagida, W. Yin and N. Yokozaki, JHEP 1804, 012 (2018) [arXiv:1801.05785 [hep-ph]]. 